\title{
ORIGINAL ARTICLE \\ Descriptive study of earthquake-related spinal cord injury in Nepal
}

\author{
CC Groves ${ }^{1,2,3}$, MK Poudel ${ }^{1}, \mathrm{M}$ Baniya ${ }^{1}, \mathrm{C} \mathrm{Rana}^{1}$ and DR House ${ }^{4,5}$
}

Study design: Descriptive study.

Objectives: To describe the epidemiological features of spinal cord injury (SCI) following the 2015 earthquakes in Nepal.

Setting: Spinal Injury Rehabilitation Centre, Kavre, Nepal.

Methods: Data were collected from the medical records of all earthquake-related patients seen from 25 April 2015 through to 16 June 2016. Data collected included patient demographics, mechanism of injury, initial medical treatment, neurological assessment, complications, neurological/functional outcomes and length of stay.

Results: Data from 117 earthquake-related SCI patients were evaluated, with a female-to-male ratio of 1.3:1. In total, 108 patients $(92 \%)$ sustained vertebral fracture and/or dislocation. Seventy-seven patients had undergone surgical fixation. The majority of patients (81\%) presented with paraplegia, of whom most $(60 \%)$ were incomplete. Thirty-eight (33\%) patients had documented pressure ulcers upon admission; six (5\%) patients developed new pressure ulcers during their rehabilitation stay. Urinary tract infection was seen in $34(29 \%)$ patients. Seven $(6 \%)$ patients were diagnosed with deep vein thrombosis. One patient developed clinically significant heterotopic ossification. Significant improvements were seen in patients' functional outcomes before discharge. Two deaths occurred in this patient population.

Conclusions: The Nepal earthquakes resulted in a significant number of $\mathrm{SCls}$, the majority occurring in women. Incomplete paraplegia was the most common presentation. Pressure ulcer, the most frequent complication, primarily occurred before rehabilitation admission. Continued efforts focused on comprehensive planning, and preparedness for SCl-specific interdisciplinary care following earthquakes, particularly in resource-limited settings, is critical to ensuring survival, preventing complications and optimizing functional outcomes in this patient population.

Spinal Cord (2017) 55, 705-710; doi:10.1038/sc.2017.25; published online 14 March 2017

\section{INTRODUCTION}

On Saturday, 25 April 2015, a 7.8-magnitude (M) earthquake struck central Nepal, causing over 8000 deaths and 21000 injuries. This was followed by hundreds of aftershocks including a notable 7.3-M aftershock on 12 May 2015 that resulted in further injury and death. The WHO (World Health Organization) estimated that during these earthquakes, more than 200 individuals sustained spinal fractures and/or spinal cord injuries. ${ }^{1,2}$

Traumatic spinal cord injury (SCI), a known consequence of earthquakes, carries high rates of morbidity and mortality. In the past decade, studies from the 7.6-M Kashmir (Pakistan) earthquake in 2005, the 8.0-M Sichuan (China) earthquake in 2008 and the 7.3-M Haiti earthquake in 2010 have contributed substantially to the knowledge base regarding earthquake-related SCI, particularly in resource-limited settings. ${ }^{3-5}$ A recent review by Gosney et al. ${ }^{6}$ highlights common features from these earthquake-related SCI studies, including increased proportion of women in comparison with studies of SCI from other causes, decreased number of cervical SCIs, higher complications in centers without rehabilitation protocols and significant challenges to conducting research in resource-limited settings.
Despite these studies, research data are still limited. Resourcelimited countries continue to face significant challenges related to SCI, particularly during large-scale disasters. With the continued high risk of earthquakes in Nepal and surrounding areas, further understanding of earthquake-related SCI is needed to continue to improve preparedness, acute post-earthquake management and ongoing rehabilitation care. Therefore, the objective of this study was to describe the epidemiologic features of earthquake-related SCI patients who presented to the Spinal Injury Rehabilitation Centre (SIRC) in Kavre, Nepal. The study not only helps us better understand earthquakerelated SCI but also helps us understand the implications for future preparedness and management related to SCI in resource-limited settings.

\section{METHODS}

Data were recorded from all earthquake-related SCI patients who presented to SIRC from April 2015 to June 2016. As data collection for the study did not begin until August 2015, earthquake-related patients who had already been discharged at that time were retrospectively enrolled. Subsequently, data were collected prospectively until the last earthquake-related patient was discharged in June 2016.

\footnotetext{
${ }^{1}$ Spinal Injury Rehabilitation Centre, Sanga, Nepal; ${ }^{2}$ Department of Physical Medicine and Rehabilitation, Indiana University School of Medicine, Indianapolis, IN, USA; ${ }^{3}$ Health, Physical, and Population Education Department, Tribhuvan University, Kathmandu, Nepal; ${ }^{4}$ Department of Emergency Medicine, Indiana University School of Medicine, Indianapolis, IN, USA and ${ }^{5}$ Department of Emergency Medicine, Patan Academy of Health Sciences, Lalitpur, Nepal

Correspondence: Dr CC Groves, Physical Medicine and Rehabilitation, Spinal Injury Rehabilitation Centre, Nasikaisthan 1, Bhainsepati, Sanga, Kavre 45210, Nepal.

E-mail: ccgroves@iupui.edu

Received 11 November 2016; revised 5 February 2017; accepted 7 February 2017; published online 14 March 2017
} 
SIRC is the largest freestanding SCI rehabilitation hospital in Nepal. Patients present from all 75 districts of Nepal, but most come from the densely populated central region. Before 2015, the average number of patients per year at this 51-SCI-bed hospital was 126 , with an average daily census of 30 . During the months following the earthquake, SIRC expanded to 150 beds. SIRC was the main hospital in Nepal to which earthquake-related SCI patients were referred for rehabilitation after initial medical evaluation and treatment. Through personal communication, we know of 24 patients who went to two other SCI rehabilitation centers in the country.

At the time of the first earthquake, SIRC's census was 38 . Within the first 3 weeks, an additional 62 patients were admitted with subsequent patients being admitted daily. Earthquake-related admissions continued for months, with the final known earthquake-related patient being admitted in March 2016. The abrupt increase in patient volume was managed by SIRC staff with the assistance of 68 foreign medical volunteers during the first 6 months post earthquake. Many local and international organizations partnered with SIRC to supply adequate and appropriate mobility devices, along with orthoses and other assistive devices and technologies.

Data collected included patient demographics (age and gender), along with data related to injuries (vertebral and associated injuries, timing and mechanism of injury), initial medical treatment, time to neurosurgical evaluation and treatment and length of acute hospitalization. Associated injuries were defined utilizing the International Spinal Cord Injury Core Data Set as 'moderate to severe traumatic brain injury, non-vertebral fractures requiring surgery, severe facial injuries affecting sense organs, major chest injuries requiring chest tube or mechanical ventilation, traumatic amputations of an arm or leg, severe hemorrhaging, or damage to any internal organ requiring surgery. ${ }^{7}$

Rehabilitation data collected included time to rehabilitation admission, International Standards for Neurological Classification of Spinal Cord Injury examinations, presence or absence of pressure ulcer on admission, complications during rehabilitation stay (new pressure ulcer, infection, deep vein thrombosis, pulmonary embolism, heterotopic ossification), bladder function and management, admission and discharge Spinal Cord Independence Measure III (SCIM III) scores, length of rehabilitation stay and location of discharge. Urinary tract infection was defined as symptomatic bacteriuria treated with antibiotics. Urodynamic assessments during the post-earthquake period were carried out selectively utilizing one-channel water cystometry ${ }^{8}$ and multichannel cystometry with urological consultation.

Data were entered into an Excel spreadsheet (Microsoft Excel for Mac, Version 14.6.3, Redmond, Washington, USA) and analyzed using Excel and SPSS (IBM SPSS Statistics for Mac, Version 23, Armonk, New York, USA). To assess functional outcomes, admission and discharge SCIM III scores were compared using the paired sample $t$-test.

\section{Statement of ethics}

We certify that all applicable institutional and governmental regulations concerning the ethical use of human volunteers were followed during the course of this research. SIRC's ethical review committee and the Nepal Health Research Council approved the study and provided oversight. The need for informed consent was waived as a significant portion of data was collected retrospectively, and all data collected were part of patients' standard medical records and were de-identified.

\section{RESULTS}

One hundred and forty-one patients were referred to SIRC with earthquake-related injuries. Of these, 24 did not have spinal cord injuries and were excluded (Figure 1). Among the remaining 117 SCI patients, $65(56 \%)$ were women with a female-to-male ratio of 1.3:1. The mean presenting age was 36.0 (s.d.=16.4), ranging from 2 to 86 years (Table 1 ).

One hundred and five of these patients (90\%) were injured during the initial April 25 earthquake, seven (6\%) sustained injuries during the May 12 earthquake and five patients (4\%) were injured during additional aftershocks. Ninety-eight (84\%) were injured when buried by rubble; 15 (13\%) fell from structures;

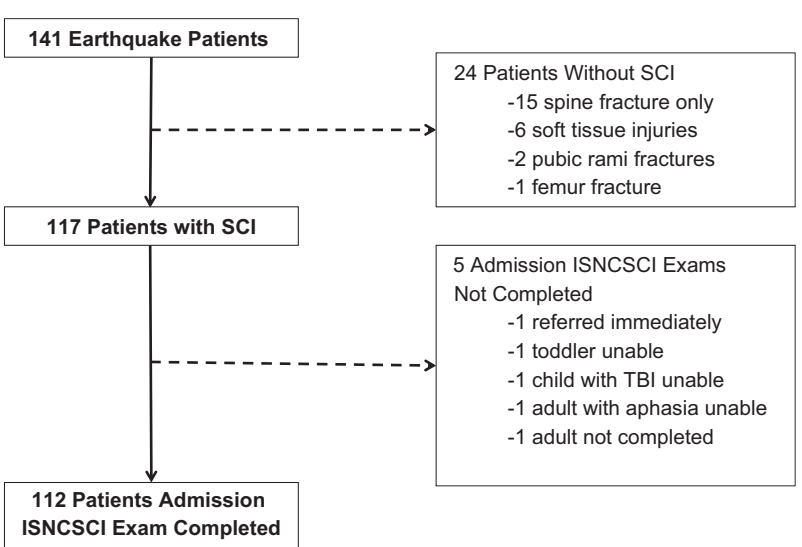

Figure 1 Patient inclusion and evaluation flow chart.

four patients (3\%) volitionally jumped from structures during the earthquakes. One hundred and eight patients (92\%) sustained vertebral fracture and/or dislocation. The majority of vertebral injuries involved the thoracolumbar junction (Table 2). Seventy-seven of these injuries were managed surgically, eight of which were determined to be unstable upon admission to SIRC and underwent surgical fixation after referral back to acute hospitals. Median time to surgery was 10 (interquartile range $=7-15$ ) days.

Associated injuries were sustained in $14(12 \%)$ patients, nine of whom sustained additional non-vertebral fractures requiring surgery with five sustaining chest injuries requiring chest tube insertion.

Time to rehabilitation varied significantly, with a range of 2-330 days post injury. Median time to rehabilitation admission was 20 (interquartile range $=11-32$ ) days. Out of the 117 SCI patients who presented to SIRC, 112 could be classified neurologically utilizing the International Standards for Neurological Classification of Spinal Cord Injury examination (Table 3). Ninety-one $(81 \%)$ of these patients presented with paraplegia, of which $47(52 \%)$ were motor incomplete (Table 4). Of the 21(19\%) patients with tetraplegia who presented, the majority were also motor incomplete (Table 5). Of note, one patient had both reported and documented lowerextremity weakness consistent with a T12 burst fracture during his acute hospitalization, but his first International Standards for Neurological Classification of Spinal Cord Injury examination was completed upon admission to rehabilitation and demonstrated intact neurology. He was given an ASIA Impairment Scale of E.

Thirty-eight $(32 \%)$ of all SCI patients had documented pressure ulcers upon admission. Five of these patients had multiple pressure ulcers. The most common location (77\%) was the sacrum (Table 6). Six patients (5\%) developed new pressure ulcers during their rehabilitation stay. Urinary tract infection was seen in $34(29 \%)$ patients. Seven $(6 \%)$ patients were diagnosed with deep vein thrombosis, whereas no patient developed clinically significant pulmonary embolism. One patient developed clinically significant heterotopic ossification. Pain and spasticity were reportedly prevalent but not routinely documented.

For bladder management, $88(75 \%)$ patients presented with indwelling urethral catheters on admission. Out of the $29(25 \%)$ patients who did not have indwelling catheters, 25 (21\%) were selfvoiding without complications and three $(2 \%)$ demonstrated urinary retention. One toddler was incontinent upon admission, but formal bladder evaluation was not performed before rapid transfer for neurosurgical evaluation. Among those who completed rehabilitation, $56(56 \%)$ were continent and self-voiding without demonstrating 
Table 1 Patient demographics

\begin{tabular}{lc}
\hline Age range, year & No. $(\%)$ of patients $(\mathrm{N}=117)$ \\
\hline $0-15$ & $4(3)$ \\
$16-30$ & $47(40)$ \\
$31-45$ & $34(29)$ \\
$46-60$ & $22(19)$ \\
$61-75$ & $9(8)$ \\
$>75$ & $1(1)$ \\
Women & $65(56)$ \\
Mean age (s.d.) & $36.0(16.4)$ \\
\hline
\end{tabular}

Table 2 Vertebral injuries

\begin{tabular}{lc}
\hline Level of injury & No. (\%) of patients $(\mathrm{N}=108)$ \\
\hline Cervical (C1-C7) & $8(7)$ \\
Thoracic (T1-T10) & $10(9)$ \\
Thoracolumbar (T11-L2) & $71(66)$ \\
Lumbar (L3-L5) & $14(13)$ \\
Sacral & $1(1)$ \\
Multi-level & $4(4)$ \\
\hline
\end{tabular}

Table 3 Presenting ISNCSCI examinations

\begin{tabular}{lc}
\hline Neurological levels of injury & No. (\%) of patients $(\mathrm{N}=112)$ \\
\hline C1-4 AIS A/B/C & $6(5)$ \\
C5-8 AIS A/B/C & $5(4)$ \\
T1-S5 AIS A/B/C & $59(53)$ \\
AIS D & $41(37)$ \\
AIS E & $1(1)$
\end{tabular}

Abbreviations: AIS, ASIA Impairment Scale; ISNCSCI, International Standards for Neurological Classification of Spinal Cord Injury.

retention or high pressures, $36(36 \%)$ patients were performing clean intermittent catheterization for bladder management, six $(6 \%)$ had indwelling urethral catheters and one utilized a suprapubic catheter.

Both admission and discharge SCIM III scores were available for 83 patients with paraplegia and for 15 patients with tetraplegia. Significant improvements were seen in SCIM III scores before discharge in both groups. For patients with tetraplegia, mean SCIM III on admission was 19.2 (s.d.=11.1) with mean discharge SCIM of 63.5 (s.d.=29.1). Mean SCIM III improvement was 44.3 (s.d. $=26.5, t=-6.46$, $P<0.001)$. For patients with paraplegia, mean SCIM III on admission was 20.3 (s.d.=16.1), with mean discharge SCIM III of 75.8 (s.d.=17.3). Mean SCIM III improvement was 55.5 (s.d.=17.9, $t=-28.28, P<0.001)$.

Among patients with paraplegia who completed rehabilitation, $55 \%$ were utilizing manual wheelchairs for mobility upon discharge, $17 \%$ utilized other mobility devices (elbow crutches, walker or cane) for ambulation, and 29\% ambulated without any device. Among patients with tetraplegia completing rehabilitation, 27\% utilized manual wheelchairs with assistance (power wheelchairs are not available in Nepal), 18\% utilized other mobility devices for ambulation and $55 \%$ ambulated without any device.
Table 4 Paraplegic ISNCSCI examinations

\begin{tabular}{lc} 
ASIA Impairment Scale (AIS) & No. (\%) of patients $(\mathrm{N}=91)$ \\
\hline AIS A & $36(40)$ \\
AIS B & $8(9)$ \\
AIS C & $15(16)$ \\
AIS D & $31(34)$ \\
AIS E & $1(1)$ \\
\hline
\end{tabular}

Abbreviations: AIS, ASIA Impairment Scale; ISNCSCI, International Standards for Neurological Classification of Spinal Cord Injury.

Table 5 Tetraplegic ISNCSCI examinations

\begin{tabular}{lcc}
\hline Neurological level of injury & No. (\%) of patients $(\mathrm{N}=21)$ & (No.) Als by level \\
\hline C1 & $1(5)$ & $(1) \mathrm{D}$ \\
C2 & $1(5)$ & $(1) \mathrm{C}$ \\
C3 & $4(19)$ & $(2) \mathrm{C},(2) \mathrm{D}$ \\
C4 & $5(24)$ & $(3) \mathrm{A},(2) \mathrm{D}$ \\
C5 & $6(28)$ & $(1) \mathrm{B},(2) \mathrm{C}, 3(\mathrm{D})$ \\
C6 & $3(14)$ & $(1) \mathrm{A},(2) \mathrm{D}$ \\
C7 & $1(5)$ & $(1) \mathrm{B}$ \\
\hline
\end{tabular}

Abbreviations: AIS, ASIA Impairment Scale; ISNCSCI, International Standards for Neurological Classification of Spinal Cord Injury.

The median rehabilitation stay was 101 (interquartile range $=45-154$ ) days. Ninety-nine patients completed inpatient SCI rehabilitation. Six patients discharged early against medical advice. Twelve patients were referred to higher levels of care and never returned to SIRC. Reasons for referral included neurosurgical consultation, surgical management of pressure ulcers, respiratory distress, delirium, sepsis and management of other active medical comorbidities including liver failure and tuberculosis. Out of the 18 patients who did not complete rehabilitation, follow-up by phone was successful in 11, two of whom died from complications related to pressure ulcers according to family reports. Both had been referred from SIRC to acute hospitals; one subsequently left from the acute hospital against medical advice, and the other patient went directly home instead of returning to SIRC. Seven of the patients who did not complete rehabilitation were unable to be reached for follow-up.

Two patients were pregnant on admission and successfully delivered full-term infants by cesarean section during their rehabilitation stays.

Given that most patients' homes were destroyed in the earthquake, safe and accessible housing was a critical need for this patient population. Of the patients who completed rehabilitation, 21 were initially discharged to a step-down facility that was built onsite for this purpose, in cooperation with a partner organization. Only 12 patients had intact homes to which they returned. The rest discharged to family members' homes, temporary shelters, rented housing or hostels.

\section{DISCUSSION}

In a country ranked as one of the most earthquake-vulnerable in the world, ${ }^{9}$ this is the first study to provide a descriptive analysis of patients with SCI following a major earthquake in Nepal. We will discuss implications surrounding particular findings related to gender, mechanism of injury, time to rehabilitation, level of injury, complications and functional outcome. We will also discuss possible mechanisms to improve disaster-related research and SCI care in Nepal. 
Table 6 Pressure ulcers by location present on admission

\begin{tabular}{lrrrrc}
\hline Stage & I & II & III & IV & Unstageable \\
\hline Sacrum & 4 & 19 & 10 & 2 & 2 \\
Trochanter & 2 & 4 & 1 & 0 & 0 \\
Ischium & 0 & 2 & 0 & 0 & 0 \\
Heel & 0 & 0 & 1 & 0 & 0 \\
Calf & 0 & 0 & 1 & 0 & 0 \\
\hline
\end{tabular}

\section{Gender}

We observed that women represented the majority of SCIs sustained during the Nepal earthquakes. This is in contrast to non-earthquakerelated traumatic SCI, in which men are more commonly injured. ${ }^{10,11}$ As was found in earthquake studies from Iran and Pakistan, the increased incidence seen in women may be related to the cultural tradition of women most often being present at home during the middle of the day, when both major earthquakes occurred., $3,6,12$ Another contributing factor may be related to the migrant work practices of men in Nepal that leave many villages with more women, increasing the likelihood of women being injured. ${ }^{13}$

This consistent finding of proportionally more female SCI patients has implications for future earthquake response and preparedness. Women often require additional devices for bladder management, which could be anticipated. Vocational training programs may need to be appropriately tailored to women within cultural contexts. Women may also represent a strategic target population for earthquake preparedness education.

\section{Mechanism of injuries}

Although the majority of injuries occurred due to structural collapse, patients also presented to SIRC due to injuries sustained by falling as well as intentionally jumping during earthquakes and aftershocks. These findings support the need for further earthquake preparedness and education in Nepal. Certainly improving the country's infrastructure with a focus on earthquake-resistant building codes is necessary for injury prevention; however, even simpler prevention campaigns such as the 'drop, cover, hold on' response to an earthquake may have adequately prevented some injuries. ${ }^{14}$ Resources that help communities develop appropriate responses during earthquake shaking are available and should be utilized. ${ }^{14}$

Of additional note, three of our presenting patients had new neurological deficits in the setting of pre-existing spinal cord injuries. Two of these patients were wheelchair users who were unable to take cover or evacuate, highlighting the reality that disabled patients are at risk for further disabling injuries during a disaster. ${ }^{15-17}$ All patients with mobility impairments should be educated regarding disaster preparedness and adequate planning to maximize their own safety in the event of a disaster requiring evacuation. ${ }^{18}$

\section{Neurological level of injury}

Higher numbers of patients with paraplegia relative to tetraplegia presented to SIRC. Certainly, the primary mechanism of injury (collapse of low walls causing hyperflexion at the thoracolumbar junction) may explain this finding. In addition, people with tetraplegia may not have survived due to prolonged times to rescue and evacuation. Rathore et al. ${ }^{3}$ suggested that no survivor with complete tetraplegia presented following the Pakistan earthquake due to complex evacuation procedures in the mountainous regions of Pakistan, which are quite similar to much of Nepal. Continued attention to rapid rescue with appropriate triage, evacuation and neurosurgical evaluation pathways is warranted to improve survival for people with tetraplegia.

In contrast to previous studies, whose reported survivors with tetraplegia ranged from 2 to $9 \%$ of all SCI patients following earthquakes, relatively more (19\%) patients with tetraplegia presented to SIRC. ${ }^{3,6,12,19,20}$ Especially in resource-limited settings, recognizing the potential for a substantial number of patients with tetraplegia provides additional challenges. Critical for this patient population is the need for trained rehabilitation professionals in respiratory management, pulmonary rehabilitation and management of the tetraplegic hand, among other aspects of rehabilitation.

\section{Complications}

The most frequent complication was pressure ulcer, $86 \%$ of which was present on admission, followed by urinary tract infection. Prevention of these complications in the SCI population requires interdisciplinary implementation of prevention strategies 'immediately' following injury. ${ }^{21,22}$ This requires having SCI-trained professionals present throughout the continuum of care, including all acute hospitals to which SCI patients are referred to for treatment.

In addition, prevention of complications requires comprehensive patient and caregiver education. ${ }^{23-26} \mathrm{We}$ found patient education to be a particularly challenging aspect of care during times of high patient volumes. As patient education requires a thorough understanding of the patient's language, social and cultural setting along with SCI-specific clinical experience/knowledge, this was one aspect of post-disaster SCI care that could not be met by foreign medical teams. Focusing on effective group education may be one strategy to consider during high patient volumes to help reduce preventable complications.

\section{Functional improvement}

Consistent with Li et al. ${ }^{19}$ evaluating earthquake-related SCI patients in China, our patients demonstrated significant functional improvement during their rehabilitation stays, as evidenced by admission/discharge SCIM III scores. Follow-up studies are needed to further assess the long-term functional outcomes of these patients.

\section{Time to rehabilitation}

Patients' time from injury to rehabilitation varied significantly, from 2 to 331 days post injury. Several factors may have contributed to the delay in referral for rehabilitation, including limited SCI awareness and knowledge about rehabilitation among the general population. Remote locations made evacuation of injured patients difficult and often resulted in delayed treatment. Additionally, tertiary hospitals were overwhelmed with patients during the initial weeks following the first earthquake, further resulting in delayed treatment.

\section{Improving spinal cord injury care in Nepal}

Prolonged times to surgery, time to rehabilitation, referrals from rehabilitation to tertiary centers for neurosurgical management and high incidence of pressure ulcers present on admission to rehabilitation highlight the need to streamline disaster response pathways for SCI patients in order to minimize complications and achieve optimal functional outcomes. Furthermore, these issues highlight the broader need in Nepal for coordinated, interdisciplinary treatment for SCI patients throughout the continuum of care. Current evidencebased guidelines recommend that SCI patients be transferred to specialized spinal injury centers as quickly as possible, as specialized care has been shown to reduce complications, reduce hospital length of stay and ultimately maximize neurological outcomes. ${ }^{22,27-29}$ 
Within the context of Nepal, we recommend the following: continuing to improve emergency medical services to ensure rapid response and safe evacuation/transfer of potential SCI patients; identification of and referral to specific acute hospitals capable of interdisciplinary SCI care immediately following injury; identification of SCI-specific rehabilitation hospitals for subsequent treatment of surgically and medically stable patients as early as possible; regular communication and coordination among involved caregivers and hospitals, including a national SCI database; and a long-term, SCI-specific follow-up system.

\section{The challenges of disaster research}

Finally, we recognize the importance of having high-quality research data from disasters, particularly in the developing world. ${ }^{30}$ However, we also recognize the significant challenges, both logistically and ethically, to progress in this area. Given that 'research in humanitarian settings should never be conducted at the expense of humanitarian action, ${ }^{31}$ we were not in a position to focus on data collection beyond the simplest medical records until three months post initial event.

Looking forward, disaster research strategies that may be appropriately considered in Nepal include the following: developing a data collection system that does not require extra staffing, reliable electricity, or internet; developing pre-approved research protocols and necessary infrastructure for the disaster setting; continuing to develop biomedical research capacity at SIRC and throughout the country; and working with SIRC's research ethics committe along with the Nepal Health Research Council and other local research ethics committees to anticipate and plan for adequate research oversight in the disaster context. $6,31,32$

\section{Limitations}

Following the disaster, data recording was limited due to overwhelming patient numbers, which increased the potential for incomplete data as well as underreporting of complications. Relatedly, there was substantial variability in the timing of International Standards for Neurological Classification of Spinal Cord Injury examinations. In addition, this study only represents those patients treated at one SCI center in Nepal. Knowing that earthquake-related spinal fracture and/or SCI patients were estimated at more than 200, data from a potentially substantial number of patients may remain unknown. Despite those estimations, we attempted to account for all earthquakerelated SCI in Nepal through contact with other facilities and believe that this data provides a reliable representation of earthquake-related SCI in Nepal.

\section{CONCLUSION}

The 2015 Nepal earthquakes resulted in a significant number of SCIs. More women were injured, incomplete paraplegia was the most common presentation, and pressure ulcer was the most common complication, primarily occurring before rehabilitation admission. Patients demonstrated significant functional gains following SCI rehabilitation. Continued efforts focusing on comprehensive planning and preparedness for SCI-specific interdisciplinary care following earthquakes, particularly in resource-limited settings, is critical to ensuring survival, preventing complications and optimizing functional outcomes in this patient population.

\section{DATA ARCHIVING}

There were no data to deposit.

\section{CONFLICT OF INTEREST}

The authors declare no conflict of interest.

\section{ACKNOWLEDGEMENTS}

We are extremely grateful for the support of many during the post-disaster period, including more than 60 international volunteers (both in person and via telephone/internet). We greatly appreciate organizational support from Asia Spinal Cord Network, Department for International Development, Direct Relief, Handicap International, International Spinal Cord Society, Médecins San Frontières, Nepal Institute of Medicine, Save the Children, Spinal Injury Sangha Nepal and the World Health Organization. Innumerable local and international donors also helped make this work possible. Particular thanks to Dr Jim Malec, Mr Stephen Muldoon, Ms. Fiona Stephenson, Dr Claire Weeks and Dr Peter Wing. Finally, we acknowledge our Nepali colleagues throughout the country who worked tirelessly to provide excellent care for thousands of patients and who recognized the need for urgent SCI-specific rehabilitation.

1 World Health Organization. Nepal earthquake situation report. Country Office for Nepal. 4 p. Report No.: 15. Available at: http://www.searo.who.int/entity/emergencies/crises/ nepal/who-sitrep15-13-may-2015.pdf (accessed on 13 May 2015).

2 World Health Organization. Nepal earthquake health cluster bulletin. 19 p. Report No.: 5. Available at: http://www.who.int/hac/global_health_cluster/countries/Health_Cluster Bulletin num5 3 June 2015.pdf?ua $=1$ (accessed on 3 June 2015).

3 Rathore MF, Rashid P, Butt AW, Malik AA, Gill ZA, Haig AJ. Epidemiology of spinal cord injuries in the 2005 Pakistan earthquake. Spinal Cord 2007; 45: 658-663.

$4 \mathrm{Li} \mathrm{Y}$, Pan F, Li Y. Analysis of rehabilitation needs, measures taken, and their effectiveness for the wounded following the Wenchuan Earthquake. J Evid Based Med 2009; 2: 258-264.

5 Burns AS, O'Connell C, Landry MD. Spinal cord injury in postearthquake Haiti: lessons learned and future needs. PM R 2010; 2: 695-697.

6 Gosney JE, Reinhardt JD, von Groote PM, Rathore FA, Melvin JL. Medical rehabilitation of spinal cord injury following earthquakes in rehabilitation resource-scarce settings: implications for disaster research. Spinal Cord 2013; 51: 603-609.

7 DeVivo M, Biering-Sørensen F, Charlifue S, Noonan V, Post M, Stripling T et al. International Spinal Cord Injury Core Data Set. Spinal Cord 2006; 44: 535-540.

8 Wyndaele JJ, THi HV, Pham BC, Kovindha A, Huong VT, Weerts E. The use of one-channel water cystometry in patients with a spinal cord lesion: practicalities, clinical value and limitations for the diagnosis of neurogenic bladder dysfunction. Spinal Cord 2009; 47: 526-530.

9 Amos J. Why Nepal is so vulnerable to quakes [Internet]. BBC News; 2015. Available at: http://www.bbc.com/news/science-environment-32462763 (accessed on 23 June 2016).

10 Ning GZ, Wu Q, Li YL, Feng SQ. Epidemiology of traumatic spinal cord injury in Asia: a systematic review. J Spinal Cord Med 2012; 35: 229-239.

11 Shrestha P, Shrestha S, Shrestha RK. Retrospective study of spinal cord injury patients admitted to spinal injury rehabilitation centre, Sanga, Banepa, Nepal. Nepal Med Coll J 2014; 16: 169-172.

12 Karamouzian S, Saeed A, Ashraf-Ganjouei K, Ebrahiminejad A, Dehghani MR, Asad AR. The neurological outcome of spinal cord injured victims of the Bam earthquake, Kerman, Iran. Arch Iran Med 2010; 13: 351-354.

13 Government of Nepal. National Population and Housing Census [Internet]. Kathmandu, NP 2011. Available at: http://cbs.gov.np/sectoral_statistics/population/national_report (accessed on 25 May 2016).

$14 \mathrm{GeoHazards}$ International. Developing Messages for Protective Action During Earthquake Shaking [Internet]. 2015. Available at: http://media.wix.com/ugd/08dab1_2079fe090f6c4a74b3110acad6a27cf1.pdf (accessed on 15 September 2016).

15 World Health Organization. Guidance note on disability and emergency risk management for health [Internet]. WHO Press; 2013. Available at: http://who.int/hac/techguidance/preparedness/disability/en/.

16 Fujii K. The Great East Japan Earthquake and disabled persons [Internet]. Japanese Society for Rehabilitation of Persons with Disabilities; 2012. Available at: http://www. dinf.ne.jp/doc/english/twg/escap_121031/fujii.html (accessed on 15 September 2016).

17 Chou YJ, Huang N, Lee CH, Tsai SL, Chen LS, Chang HJ. Who is at risk of death in an earthquake? Am J Epidemiol 2004; 160: 688-695.

18 McClure LA, Boninger ML, Oyster ML, Roach MJ, Nagy J, Nemunaitis G. Emergency evacuation readiness of full-time wheelchair users with spinal cord injury. Arch Phys Med Rehabil 2011; 92: 491-498.

19 Li Y, Reinhardt JD, Gosney JE, Zhang X, Hu X, Chen S et al. Evaluation of functional outcomes of physical rehabilitation and medical complications in spinal cord injury victims of the Sichuan earthquake. J Rehabil Med 2012; 44: 534-540.

20 Rauch A, Baumberger M, Moise FG, von Elm E, Reinhardt JD. Rehabilitation needs assessment in persons with spinal cord injury following the 2010 earthquake in Haiti: a pilot study using an ICF-based tool. J Rehabil Med 2011; 43: 969-975. 
21 Consortium for Spinal Cord Medicine Clinical Practice Guidelines. Pressure ulcer prevention and treatment following spinal cord injury: a clinical practice guideline for health-care professionals. J Spinal Cord Med 2001; 24 (Suppl 1): S40-S101.

22 Consortium for Spinal Cord Medicine Clinical Practice Guidelines. Early acute management in adults with spinal cord injury: a clinical practice guideline for health-care professionals [Internet]. Paralyzed Veterans of America; 2008. Available at: http://www. ncbi.nlm.nih.gov/pmc/articles/PMC2582434/ (accessed on 15 September 2016).

23 Hsieh JMA, Iruthayarajah J, Loh E, Ethans K, Mehta S, Wolfe D et al. Bladder management following spinal cord injury [Internet]. Spinal Cord Injury Research Evidence. 2014. Available at: http://www.scireproject.com/sites/default/files/bladder_management.pdf (accessed on 12 September 2016).

24 Hsieh JMA, Wolfe D, Lala D, Titus L, Campbell K, Teasell R. Pressure ulcers following spinal cord injury [Internet]. Spinal Cord Injury Research Evidence. 2014. Available from http://www.scireproject.com/sites/default/files/pressure_ulcers.pdf (accessed on 12 September 2016).

25 Kristofer Hagglund MC, Schopp L, Sherman A, Acuff M. Consumer-assistant education to reduce the occurrence of urinary tract infections among persons with spinal cord injury. Top Spinal Cord Inj Rehab 2005; 10: 53-62.
26 Gelis A, Stefan A, Colin D, Albert T, Gault D, Goossens D et al. Therapeutic education in persons with spinal cord injury: a review of the literature. Ann Phys Rehabil Med 2011; 54: 189-210.

27 Wolfe D, Hsieh JTC, Mehta S. Rehabilitation practices and associated outcomes following spinal cord injury [Internet]. Spinal Cord Injury Research Evidence. 2010. Available at: http://www.scireproject.com/rehabilitation-evidence/rehabilitation-practices/what-sci-rehabilitation (accessed on 4 September 2016).

28 Scivoletto G, Morganti B, Molinari M. Early versus delayed inpatient spinal cord injury rehabilitation: an Italian study. Arch Phys Med Rehabil 2005; 86: 512-516.

29 Pagliacci MC, Celani MG, Zampolini M, Spizzichino L, Franceschini M, Baratta S et al. An Italian survey of traumatic spinal cord injury. The Gruppo Italiano Studio Epidemiologico Mielolesioni study. Arch Phys Med Rehabil 2003; 84: 1266-1275.

30 Roy N, Thakkar P, Shah H. Developing-world disaster research: present evidence and future priorities. Disaster Med Public Health Prep 2011; 5: 112-116.

31 Eckenwiler L, Pringle J, Boulanger R, Hunt M. Real-time responsiveness for ethics oversight during disaster research. Bioethics 2015; 29: 653-661.

32 O'Mathuna D. Research ethics in the context of humanitarian emergencies. J Evid Based Med 2015; 8: 31-35. 